\title{
GRB as luminosity indicator
}

\author{
Rupal Basak and A. R. Rao \\ Tata Institute of Fundamental Research, Mumbai
}

\begin{abstract}
Gamma Ray Bursts (GRBs) are found at much higher redshifts ( $\mathrm{z}>6$ ) than Supernova Ia $(\mathrm{z} \sim 1)$, and hence, they can be used to probe very primitive universe. However, radiation mechanism of GRB remains a puzzle, unlike Supernova Ia. Through comprehensive description, both empirical and physical, we shall discuss the most likely way to use the constituent pulses of a GRB to find the radiation mechanism as well as using the pulses as luminosity indicators.
\end{abstract}

Keywords. gamma-ray burst - general , method — data analysis, radiation mechanism thermal and non-thermal, cosmology — luminosity indicator

\section{Introduction}

First discovered during late 1960's, Gamma Ray Burst (GRB) soon became one of the greatest puzzles in astrophysics in terms of its location, size and energetics. Many satellites have been flown since then to understand this puzzling phenomenon - HETE2, BATSE, Swift, Fermi, to name a few. Though we have a clear idea about their cosmic origin (the highest spectroscopic redshift being 8.2 for GRB 090423) and a rough idea about their energy budget (highest among all astrophysical phenomena $-\sim 10^{52} \mathrm{erg}$ ), the emission mechanism is still unsettled. Hence, in spite of a great hope that GRB could be used as luminosity indicator in extension to the currently used ones, e.g., supernova Ia, one has to standardize the GRB energetics first. This, of course, serves two purposes — solving the GRB physics, which is not settled till now, and using GRB as luminosity indicator.

\section{Methodology and Results}

There exist certain empirical correlations of the peak energy $\left(E_{\text {peak }}\right)$ of GRB spectrum with the energetics of GRB. These correlations are important as they can be used to independently measure a physical parameter, namely energy, using only prompt emission spectral data. Amati et al. (2002) showed that $E_{\text {peak }}$ correlates with the isotropic equivalent energy $\left(E_{\gamma, i s o}\right)$. It is very important that this correlation should hold within a GRB, as that can prove the reality of such correlation and strongly refutes selection bias. But, Basak \& Rao (2012b), using 9 GRBs with known redshift detected by Fermi/Gamma Ray Burst Monitor (GBM), have shown that this correlation breaks down if one uses the time-resolved data. The Pearson correlation, 0.80 drops to 0.37 . They concluded that Amati correlation has no meaning in a time-resolved study. The situation is saved if one uses the constituent broad pulses (total 22 pulses), rather than intensity guided time cuts. Pulse-wise analysis not only restores the correlation, it improves that (0.89). They used the pulse description of Basak \& Rao (2012a) and found that replacement of $E_{\text {peak }}$ with a new quantity of their model, namely the peak energy at zero fluence $\left(E_{\text {peak }, 0}\right)$ improves the correlation even further (0.96).

In this study, we have enlarged our sample to 19 GRBs (43 pulses) having measured redshifts $(\mathrm{z})$ from GBM catalog. We consider only pulse-wise Amati correlation here. The 
Basak, R. \& Rao, A. R.

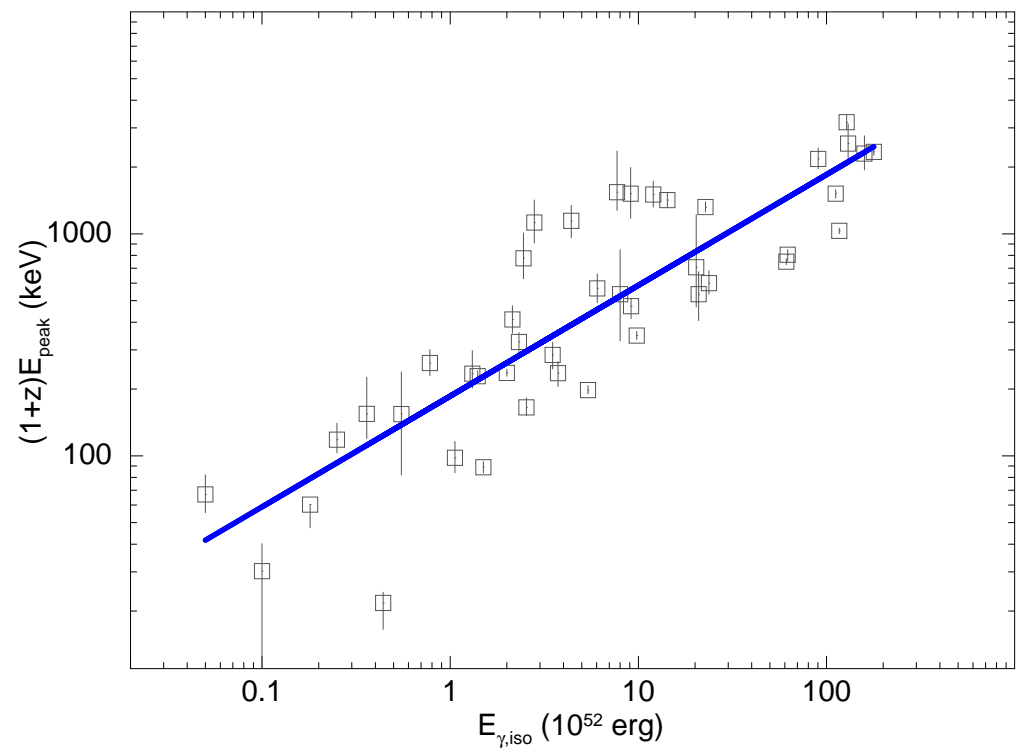

Figure 1. Pulse-wise Amati correlation. The thick line shows the correlation

Spearman rank correlation coefficient is 0.86 (see Figure 1). As we have a larger sample, we are able to divide the set of GRBs into various redshift (z) bins and study evolution of the correlation. A detailed analysis will be published later.

\section{Discussions}

The prompt emission spectrum of a GRB is generally fitted with Band model (Band et al. 1993). The empirical correlations, we have discussed, relies on the fact that the overall and instantaneous spectrum is Band like. Band model describes a non-thermal model. There are alternative models, e.g., black body with a powerlaw (Ryde 2004). Recently, we have analyzed the brightest GRBs, having separable pulses, namely GRB 081221 and GRB 090618, and found that the Band model is adequate in the falling part of a pulse. But, a different model is preferred in the rising part (Basak \& Rao 2013). Hence, one should take into account these findings while describing the emission mechanism of GRB and thereby using the pulses for cosmological purpose.

\section{References}

Amati, L., Frontera, F., Tavani, M., in’t Zand, J. J. M., Antonelli, A., Costa, E., Feroci, M., Guidorzi, C., Heise, J., Masetti, N., Montanari, E., Nicastro, L., Palazzi, E., Pian, E., Piro, L., Soffitta, P. 2002, A\& A, 390, 81

Band, D., Matteson, J., Ford, L., Schaefer, B., Palmer, D., Teegarden, B., Cline, T., Briggs, M., Paciesas, W., Pendleton, G., Fishman, G., Kouveliotou, C., Meegan, C., Wilson, R., Lestrade, P. 1993, ApJ, 413, 281

Basak, R., Rao, A. R. 2012a, ApJ, 745, 76

Basak, R., Rao, A. R. 2012b, ApJ, 749, 132

Basak, R., Rao, A. R. 2013, arXiv: 1302.6091

Ryde, F. 2004, ApJ, 614, 827 